antibodies bind to gp120, an HIV surface protein that the virus uses to enter host cells. A subset of the antibodies neutralized $96 \%$ of 118 viruses in a test panel. Although the antibodies were highly mutated, they all shared a sequence of 68 amino acids. Science doi:10.1126/ science.1207227 (2011)

\section{VACCINES \\ Mix-and-match for meningitis}

A vaccine for meningitis has long eluded researchers because the key antigen of the meningococcus $B$ strain has more than 300 sequence variations. Using the three-dimensional crystal structure of this antigen, fHBP, researchers have engineered an antigen that carries the main amino-acid variants and elicits antibodies against all strains of the bacterium in mice.

Rino Rappuoli at Novartis Vaccines and Diagnostics in Siena, Italy, Lucia Banci at the University of Florence, Italy, and their co-workers analysed the sequences of the 300 different types of fHBP. They classified the variants into three main groups and then engineered variants from one group to carry sequences from the other two. They tested 54 engineered fHBPs in mice and one stood out for its ability to induce the production of bacterium-killing antibodies. The authors say it could be used in a vaccine and that the approach could aid in the design of vaccines for other pathogens with many natural variants.

Sci. Transl. Med. 3, 91ra62 (2011)

\section{DNA-inspired polymerization}

Polymerization typically relies on harmful metal catalysts, but researchers in Japan have succeeded in circumventing this problem. Akira Harada at Osaka University and his colleagues constructed a synthetic polymerase that can catalyse the synthesis of highmolecular-weight polymers.

The polymerase is made up of two ring-shaped sugar molecules called cyclodextrins (CDs) linked together by a flexible covalent chain. The authors propose that one of the CDs functions as the active site, where key bonds in an incoming cyclic ester monomer are broken to open up the ester's ring, allowing it to bond with other monomers and form a chain. The second CD functions as a clamp, threading the growing chain through its hollow structure to hold the chain in place. As the polymerization proceeds, the growing chain slides by one position, freeing up space for the next incoming monomer.

The structure's design was inspired by the polymerases that synthesize DNA.

Angew. Chem. Int. Edn

doi:10.1002/anie.201102834 (2011)

\section{CANCER}

\section{Tissues stretch to let tumours move}

A protein made by connectivetissue cells causes mechanical changes in tissue structure that help cancers to spread around the body.

Jacky Goetz and Miguel

Del Pozo at the Spanish

National Center for

Cardiovascular Research in Madrid and their colleagues found that stromal fibroblast cells surrounding many human cancers express high levels of the protein CAV1. Mouse fibroblasts expressing CAV 1 activate the enzyme Rho, which causes the cells to stretch out (pictured). In three-dimensional gel matrices in vitro, the elongated fibroblasts formed stiff, parallel-fibre networks through which cancer cells moved rapidly.

When the authors injected mouse fibroblasts lacking CAV1 and breast-cancer cells into mice, tumours were

COMMUNITY CHOICE

The most viewed papers in science

MATERIALS

\title{
Graphene textiles for energy storage
}

on HighLY READ
in pubs.acs.org
in June

Porous textiles coated with atom-thick sheets of carbon called graphene could underpin cheap and long-lasting energystorage systems.

Zhenan Bao, Yi Cui and their colleagues at Stanford University in California dipped polyester fibres into a graphene solution and then deposited manganese dioxide onto the resulting structure. They used this as an electrode, combined with another made from carbon-nanotubecoated textiles, in a sodium sulphate solution. The resulting supercapacitor maintained a high level of energy storage and power delivery over 5,000 charge and discharge cycles, which is unusually long-lasting for manganese-dioxide-based electrodes. The fibres' three-dimensional porous structure has a larger surface area than conventional electrodes, enhancing performance.

Moreover, the system is made from abundant and environmentally friendly materials using a scalable process, the researchers say.

Nano Lett. doi:10.1021/nl2013828 (2011)

minimally invasive. But when they used CAV1-expressing fibroblasts, tumours grew more rapidly and invaded multiple organs.

Cell doi:10.1016/j. cell.2011.05.040 (2011)

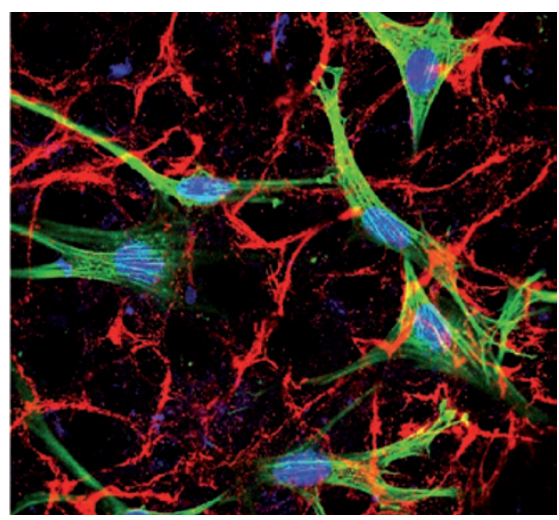
PALAEONTOLOGY

\section{Bridging the dino gap}

Did an asteroid impact end the dinosaurs' reign? The controversy surrounding this question has been driven by a lack of dinosaur fossils dating to the period leading up to the impact in question.
Now researchers show that a fossilized dinosaur horn found in Montana is from the relevant period, suggesting that dinosaurs were not extinct before the impact.

Tyler Lyson at Yale

University in New Haven, Connecticut, and his colleagues found a 45-centimetrelong ceratopsian brow horn $13 \mathrm{~cm}$ below the Cretaceous-Tertiary boundary, a geological feature thought to mark the time of the extraterrestrial impact in what is now Mexico around 65 million years ago. They identified the boundary through analysis of nearby rocks and Cretaceous fossils. The horn is the youngest non-avian dinosaur fossil yet discovered.

Biol. Lett. doi:10.1098/ rsbl.2011.0470 (2011) For a longer story on this research, see go.nature.com/ $\mathrm{kx} 56 \mathrm{ec}$

\section{$\rightarrow$ NATURE.COM}

For the latest research published by Naturevisit:

www.nature.com/latestresearch 